\title{
Reação de clones de batata a Alternaria solani em condições de campo
}

\section{Reaction of clones of potato to Alternaria solani under field conditions}

\author{
Patrícia Mayumi OKITA ${ }^{1}$; Camila Vilela VASCONCELOS ${ }^{2}$; Igor Pereira da COSTA ; $^{3}$ \\ Nei PEIXOTO ${ }^{4}$; Daniel Diego Costa CARVALHO ${ }^{5}$ \\ ${ }^{1}$ Graduação em Agronomia; Universidade Estadual de Goiás - Unidade Ipameri; \\ patriciamayumiagro@yahoo.com.br \\ ${ }^{2}$ Mestranda em Produção Vegetal; Universidade Estadual de Goiás - Unidade Ipameri; \\ camilavilela85@hotmail.com; \\ ${ }^{3}$ Graduação em Agronomia; Universidade Estadual de Goiás - Unidade Ipameri; \\ igorpereiraagronomia@yahoo.com.br \\ ${ }^{4}$ Prof. Dr.; Universidade Estadual de Goiás - Unidade Ipameri; nei.peixoto48@gmail.com \\ ${ }^{5}$ Autor para correspondência; Prof. Dr.; Universidade Estadual de Goiás - Unidade Ipameri; Laboratório de \\ Fitopatologia; Rodovia GO 330, km 241, Anel Viário, Setor Universitário, CEP 75780-000, Ipameri, GO, Brasil; \\ daniel.carvalho@ueg.br
}

Recebido em: 11-12-2013; Aceito em: 02-04-2014

\begin{abstract}
Resumo
A batata é o terceiro alimento mais importante para a humanidade, possuindo elevado potencial produtivo. No entanto, a doença conhecida como pinta-preta é um dos principais fatores limitantes, podendo causar perdas de 6 a 100\% na produção. O objetivo deste trabalho foi selecionar clones de batata com resistência à pinta-preta. O experimento de campo foi conduzido no ano de 2010, em delineamento em blocos casualizados (DBC), com 10 tratamentos e quatro repetições (parcelas de $32 \times 14 \mathrm{~m}$ ). Foram utilizados nove clones e a cultivar 'Asterix' como padrão de resistência. As avaliações de severidade da doença foram realizadas aos 70; 85; 95 e 105 dias após a emergência (DAE), e a colheita, aos $120 \mathrm{DAE}$. Os dados referentes à severidade e à produtividade média de tubérculos por hectare foram submetidos à análise de variância e ao teste de Scott-Knott $(P \leq 0,05)$; além disso, modelos de regressão linear foram obtidos para estimar a taxa de progresso da pinta-preta da batata, dos 70 aos 105 DAE. Os clones CNPH CIP 53 e CNPH CIP 44 apresentaram valores de severidade aos $105 \mathrm{DAE}$, de $18,5 \%$ e $34,25 \%$, respectivamente, sendo estatisticamente inferiores aos demais tratamentos, os quais variaram entre 42 e $70 \%$ de área da parcela coberta com os sintomas de pinta-preta. Com base nos resultados obtidos, conclui-se que os clones CNPH CIP 53 e CNPH CIP 44 são os dois melhores clones quanto à resistência à pinta-preta, e os modelos de regressão linear são bem ajustados para estimar a taxa de progresso da pinta-preta da batata a partir dos 70 DAE.
\end{abstract}

Palavras-chave adicionais: epidemiologia de doenças de plantas; melhoramento genético; pintapreta; tubérculo.

\begin{abstract}
Potato is the third more important food for mankind. However, the disease known as early blight is one of the most important limiting factor capable of yield reductions between 6 and $100 \%$. The objective of this study was to select potato clones showing resistance to early blight. The experiment was carried out in the field during the crop year of 2010 with ten treatments and four repetitions which were distributed in the field according to a randomized complete block design. Each plot had an area of $32 \times 14 \mathrm{~m}$. Nine potato clones and one cultivar ('Asterix') were evaluated. The evaluations of resistance to disease were conducted $70,85,95$, and 105 days after emergence (DAE) and harvest was made $120 \mathrm{DAE}$. The experimental data were submitted to the analysis of variance and the means compared by the Scott-Knott $(P<0.05)$ test. In addition to that, linear regression models were obtained to estimate the rate of progress of early blight between 70 and 195 DAE. Clones CNPH CIP 53 and CNPH CIP 44 showed severity of disease values at 105 DAE of 18.5 and $34.25 \%$ respectively. The statistical analysis showed these values to be significantly inferior to those shown by the other clones. So, clones CNPH CIP 53 and CNPH CIP 44 are indicated as resistant to early blight disease. The linear regression models are well adjusted to estimate the rate of progress of early blight starting 70 DAE.
\end{abstract}

Additional keywords: early blight; epidemiology of plant diseases; genetic breeding; tuber. 


\section{Introdução}

A batata (Solanum tuberosum L.) é o terceiro alimento mais importante para a humanidade, em termos de consumo humano, ficando atrás somente do arroz e do trigo, com produção mundial de mais de 324 milhões de toneladas, sendo que somente a China $(74,8$ milhões de toneladas) e a Índia (36,6 milhões de toneladas) são responsáveis por um terço dessa produção (FAO, 2013). A safra brasileira de batata, em 2013, foi estimada em 3.528.071 toneladas, sendo Minas Gerais o principal produtor, seguido por Paraná, São Paulo e Rio Grande do Sul (IBGE, 2013). Consumida nas mais variadas formas, essa solanácea possui grande importância econômica, social e cultural, graças a sua ampla adaptabilidade a diferentes agroecossistemas e elevado potencial produtivo; sendo assim, é a olerácea com a maior área cultivada no Brasil (TÖFOLI et al., 2013).

Embora possua elevado potencial produtivo, a cultura da batata é acometida por mais de 100 doenças bióticas causadas por diferentes espécies de fungos, bactérias, nematoides e vírus (NEDER et al., 2010). Entre as doenças fúngicas mais importantes, em especial para as condições tropicais, está a pinta-preta, causada pelo fungo Alternaria solani (SIMON et al., 2009). Sob temperatura e umidade elevadas, esta doença é um dos principais fatores limitantes do cultivo na época das águas, podendo causar danos de 6 a 100\% na produção, caso não sejam realizadas pulverizações ou as epidemias se iniciem de forma precoce (TÖFOLI et al., 2013). A doença é caracterizada pela formação de lesões castanho-escuras e necrose do tecido entre as nervuras da folha (SHAHBAZI et al., 2011). Entre os fatores que contribuem para a ocorrência de epidemias severas de pinta-preta, destacam-se: plantio massivo de clones suscetíveis, predominância de condições climáticas favoráveis no decorrer das principais safras, alta capacidade de esporulação do agente causal, presença de hospedeiros intermediários, existência de inóculo no decorrer do ano inteiro e facilidade de disseminação (TÖFOLI et al., 2013).

A técnica mais utilizada pelos agricultores para o controle da pinta-preta consiste na aplicação de fungicidas; entretanto, estes causam poluição ambiental e aumento na necessidade de mão de obra, além de muitas vezes não controlarem o patógeno (JANSKY et al., 2008; NEDER et al., 2010). No Brasil, os fungicidas são pulverizados periodicamente, e mais de oito aplicações são usadas por ciclo de cultivo, representando mais de $10 \%$ dos custos totais de produção (DITA RODRÍGUEZ et al., 2006; RODRIGUEZ et al., 2007). Portanto, o uso de variedades resistentes torna-se a alternativa mais viável a longo prazo.

Trabalhos vêm sendo realizados no Brasil com o objetivo de desenvolver genótipos resistentes à pinta-preta, destacando-se 0 de NEDER et al. (2010), que avaliaram 331 clones de batata, dos quais 20 foram selecionados quanto ao desempenho agronômico e resistência à pinta-preta e ao vírus PVY e PVX. De forma semelhante, SIMON et al. (2009) selecionaram genótipos que associam alto nível de resistência à pinta-preta e tolerância ao calor. Desta forma, o presente trabalho objetivou selecionar clones de batata com resistência à pinta-preta.

\section{Material e métodos}

O experimento foi conduzido em campo, durante o período de 28-08-2010 a 30-12-2010, em área experimental da Universidade Estadual de Goiás, Unidade Ipameri, Goiás, Brasil, cujas

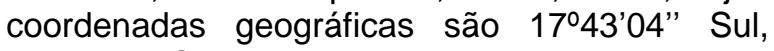
$48^{\circ} 08^{\prime} 43^{\prime \prime}$ Oeste e altitude de $794 \mathrm{~m}$.

O solo da área experimental foi classificado como Latossolo Vermelho-Amarelo distrófico, com pH de 5,8. Conforme análise de solo, estimaram-se os teores de $\mathrm{Ca}, \mathrm{Mg}, \mathrm{Al}$ e $\mathrm{H}+\mathrm{Al}$, em 13,$4 ; 7,9 ; 0,31$ e $25,1 \mathrm{mmol}_{\mathrm{c}} \mathrm{dm}^{-3}$; para $\mathrm{P}$ e K foram encontrados $2,4 \mathrm{mg} \mathrm{dm}^{-3} \mathrm{e} 1,68 \mathrm{mmol}_{\mathrm{cm}} \mathrm{dm}^{-3}$, respectivamente, e, quanto à matéria orgânica, $21 \mathrm{~g} \mathrm{dm}^{-3}$.

O preparo do solo foi do tipo convencional, com aração seguida de nivelamento, e as covas foram abertas por sulcagem. A adubação de plantio foi feita conforme recomendação de adubação de batata para a região, sendo utilizados $3.000 \mathrm{~kg} \mathrm{ha}^{-1}$ do formulado NPK 05-25-15, jogados no sulco de plantio.

O delineamento experimental utilizado foi o em blocos casualizados (DBC), com 10 tratamentos e quatro repetições, sendo que cada parcela possuía quatro fileiras contendo dez plantas cada uma, dispostas no espaçamento de $0,80 \times 0,35 \mathrm{~m}$, sendo utilizadas para as avaliações apenas as 20 plantas centrais.

Foram avaliados nove clones de batata (CNPH CIP 013, CNPH CIP 015, CNPH CIP 43, CNPH CIP 44, CNPH CIP 53, CNPH CIP 54, CNPH CIP 91, CNPH CIP 103 e CNPH CIP 110), pré-selecionados a partir do programa de melhoramento da Embrapa-Hortaliças (PEIXOTO et al., 2002), e a cultivar Asterix como testemunha.

O sistema de irrigação utilizado foi do tipo gotejamento, e a amontoa foi realizada aos 30 dias após a emergência das plantas (DAE), quando elas estavam com altura média de $30 \mathrm{~cm}$, antes da qual foi feita adubação de cobertura com $300 \mathrm{~kg} \mathrm{ha}^{-1}$ de sulfato de amônia. O controle de plantas daninhas deu-se através de capina manual, sendo os demais tratos 
sanitários limitados apenas ao controle de pragas.

Aos 70; 85; 95 e 105 DAE (10-11-2010, 25-11-2010, 05-12-2010 e 15-12-2010), respectivamente, realizou-se a avaliação da severidade de pinta-preta nas plantas de batata, mediante 0 emprego de escala decritiva de notas, de acordo com a porcentagem de área foliar da parcela que exibiu sintomas da doença (adaptada de REIFSCHNEIDER et al., 1984), sendo elas: Nota 1 - parcela com plantas sem sintomas; Nota 2 - até 2,5\% de área foliar da parcela coberta por sintomas (afpcs); Nota 3 - de 2,6 até $12 \%$ de afpcs; Nota 4 - de 13 até $25 \%$ de afpcs; Nota 5 - de 26 até $50 \%$ de afpcs; Nota 6 - de 51 até $75 \%$ de afpcs, e Nota 7 - de 76 até $100 \%$ de afpcs.

A colheita foi realizada aos 120 DAE, quando as hastes estavam completamente secas, e os tubérculos, com a película firme. Os dados referentes à severidade e à produtividade média de tubérculos por hectare foram submetidos à análise de variância, ao teste de ScottKnott $(P \leq 0,05)$. As análises de regressão para a severidade foram realizadas no programa estatístico SISVAR (FERREIRA, 2011). Para as análises estatísticas, foi considerado o ponto médio de cada nota atribuída, o que permitiu expressar os resultados em porcentagem de área avaliada de plantas com sintomas de pinta-preta.

\section{Resultados e discussão}

Após análise dos dados, verificou-se que os clones CNPH CIP 53 e CNPH CIP 44 foram superiores aos demais clones (18,5\% e 34,25\%, respectivamente), pois apresentaram valores de severidade aos 105 DAE, estatisticamente inferiores aos demais tratamentos (Tabela 1). Os genótipos CNPH CIP 43, CNPH CIP 54, CNPH CIP 110, CNPH CIP 103, Asterix, CNPH CIP 91, CNPH CIP 013 e CNPH CIP 015 foram os oito mais suscetíveis ao fungo $A$. solani em condições de campo, apresentando valores de severidade que variaram de 42 até $70 \%$ de área da parcela coberta com os sintomas de pinta-preta.

PINTO et al. (2002) sugerem a avaliação da gravidade da doença, no auge do desenvolvimento da epidemia, considerando que a ação dos componentes de resistência é cumulativa e eles agem juntos. Com base nessa premissa é que se considerou, para o teste de médias, as leituras realizadas aos 105 DAE. No presente trabalho, foram observadas diferenças significativas entre os clones CNPH CIP 53 e CNPH CIP 44 versus testemunha (Tabela 1 ). Tais resultados estão em conformidade com NEDER et al. (2010), que também verificaram diferença significativa entre os tratamentos quanto à resistência à pinta-preta, evidenciando a variabilidade de resposta à infecção com o fungo Alternaria solani e comprovando a existência de resistência genética. De forma semelhante a NEDER et al. (2010), SIMON et al. (2009) observaram diferenças significativas para resistência à pinta-preta em experimento conduzido na presença de inoculação do patógeno. Um ponto de destaque do presente trabalho é que, diferentemente de NEDER et al. (2010) e SIMON et al. (2009), não houve a necessidade de inoculação de $A$. solani, o que faz os dados de severidade de pinta-preta, bem como os modelos de regressão (Tabela 1) se aproximarem das reais condições de cultivo.

Tabela 1. Severidade da pinta-preta aos 105 dias após a emergência (DAE), modelos lineares de regressão e produtividade de tubérculo dos dez genótipos de batata avaliados em campo experimental. Severity of early blight 105 DAE, linear regression models and tuber productivity of ten potato genotypes.

\begin{tabular}{|c|c|c|c|c|c|}
\hline Clones de batata & $\begin{array}{c}\text { Severidade aos } 105 \\
\operatorname{DAE}^{(1,2)}\end{array}$ & Modelo linear & $\mathrm{R}^{2}(\%)$ & $(P \leq X)$ & $\begin{array}{l}\text { Produtividade } \\
\left(\mathrm{kg} \mathrm{ha}^{-1}\right)^{(\mathrm{ns})}\end{array}$ \\
\hline$\overline{\mathrm{CNPH}}$ CIP 53 & $18,50 \mathrm{a}$ & $y=0,3874 x-21,2942$ & 88,59 & 0,01 & 5161 \\
\hline CNPH CIP 44 & 34,25 a & $y=0,9088 x-63,2879$ & 90,83 & 0,01 & 4641 \\
\hline CNPH CIP 43 & $42,25 \mathrm{~b}$ & $y=1,1484 x-80,8808$ & 87,11 & 0,01 & 5548 \\
\hline CNPH CIP 54 & $50,00 \mathrm{~b}$ & $y=1,2639 x-89,9369$ & 84,82 & 0,01 & 3878 \\
\hline CNPH CIP 110 & $50,00 \mathrm{~b}$ & $y=1,2230 x-81,1518$ & 91,14 & 0,01 & 5907 \\
\hline CNPH CIP 103 & $51,50 \mathrm{~b}$ & $y=0,9834 x-54,8714$ & 95,59 & 0,05 & 4336 \\
\hline Asterix & $54,16 \mathrm{~b}$ & $y=0,9738 x-54,1083$ & 84,81 & 0,01 & 4855 \\
\hline CNPH CIP 91 & $57,75 b$ & $y=1,4821 x-97,0418$ & 95,54 & 0,01 & 3781 \\
\hline CNPH CIP 013 & $62,50 \mathrm{~b}$ & $y=1,3515 x-84,4941$ & 94,15 & 0,01 & 3583 \\
\hline CNPH CIP 015 & $70,83 \mathrm{~b}$ & $y=1,7899 x-134,386$ & 71,26 & 0,01 & 6188 \\
\hline$\overline{\mathrm{CV}}(\%)$ & 22,60 & & & & 25,43 \\
\hline
\end{tabular}

Após análise de regressão, a severidade da doença dos tratamentos foi ajustada por mo- delos lineares simples, significativos $(P \leq 0,05)$, e com coeficiente de determinação $\left(R^{2}\right)$ acima de 
$71,26 \%$ (Tabela 1). Os coeficientes de variação (CV) para severidade, aos $105 \mathrm{DAE}$, e para produtividade foram de $22,60 \%$ e $25,43 \%$, respectivamente, os quais são considerados altos de acordo com PIMENTEL GOMES (2000). No entanto, estes foram menores que os encontrados por outros autores, uma vez que valores de CVs altos são comuns em experimentos de batata (SIMON et al., 2009).

Não houve diferença estatística entre os dados de produtividade dos tratamentos, os quais variaram de 3.583 a $6.188 \mathrm{~kg} \mathrm{ha}^{-1}$. A baixa produtividade obtida no experimento pode ser explicada pela não utilização de produtos químicos para o controle de pragas e doenças com vistas a identificar resistência, principalmente à pinta-preta. Assim, em condições ótimas de cultivo, o experimento poderia ter sua produtividade elevada. Segundo FILGUEIRA (2008), a média nacional de produtividade é de $20.000 \mathrm{~kg} \mathrm{ha}^{-1}$. O manejo adotado no presente estudo equipara-se ao orgânico, em conformidade com MAGGIO et al. (2008), que reportaram redução de $25 \%$ da produtividade do sistema orgânico de produção em relação ao convencional. Assim, como a cultura da batata é muito sensível à não utilização de insumos, considera-se que este tenha sido o fator determinante para a redução da produtividade. NEDER et al. (2010) também obtiveram diferenças significativas entre os clones de batata quanto à resistência à pinta-preta $e$, de forma semelhante ao estudo em questão (Tabela 1), as médias de produção também foram baixas, cerca de $11.700 \mathrm{~kg} \mathrm{ha}^{-1}$, em decorrência da incidência de requeima (Phytphthora infestans) no experimento.

No caso presente, as curvas de progresso da doença foram construídas para o patossistema estudado no experimento, resumindo a relação entre doença e tempo. Assim, na Figura 1, pode-se notar que o clone CNPH CIP 53, apesar de aos 85 DAE ter uma pequena elevação na severidade, manteve-se praticamente estável em todo o ciclo, não permitindo o aumento da taxa de progresso da doença. Já a clone CNPH CIP 44 permitiu severidade menor que o clone CNPH CIP 53 até os 85 DAE; no entanto, após esta data, houve um acréscimo contínuo na severidade. Entretanto, os dois clones (CNPH CIP 53 e CNPH CIP 44) foram estatisticamente similares, aos $105 \mathrm{DAE}$, e inferiores aos demais quanto à severidade de pinta-preta (Tabela 1). Diferentemente destes dois clones, 0 CNPH CIP 015 apresentou a severidade baixa até os $95 \mathrm{DAE}$, sendo que após esta data houve um elevado aumento na severidade (Figura 1). $O$ clone CNPH CIP 54, de forma semelhante aos três clones citados anteriormente, manteve-se estável até os 95 DAE; no entanto, após esta data, aumentou significativamente a severidade de pinta-preta. Os clones CNPH CIP 110, CNPH CIP 91, CNPH CIP 013, CNPH CIP 103 e Asterix, já aos $70 \mathrm{DAE}$, apresentaram alta severidade da doença em comparação aos outros clones (Figura 1).

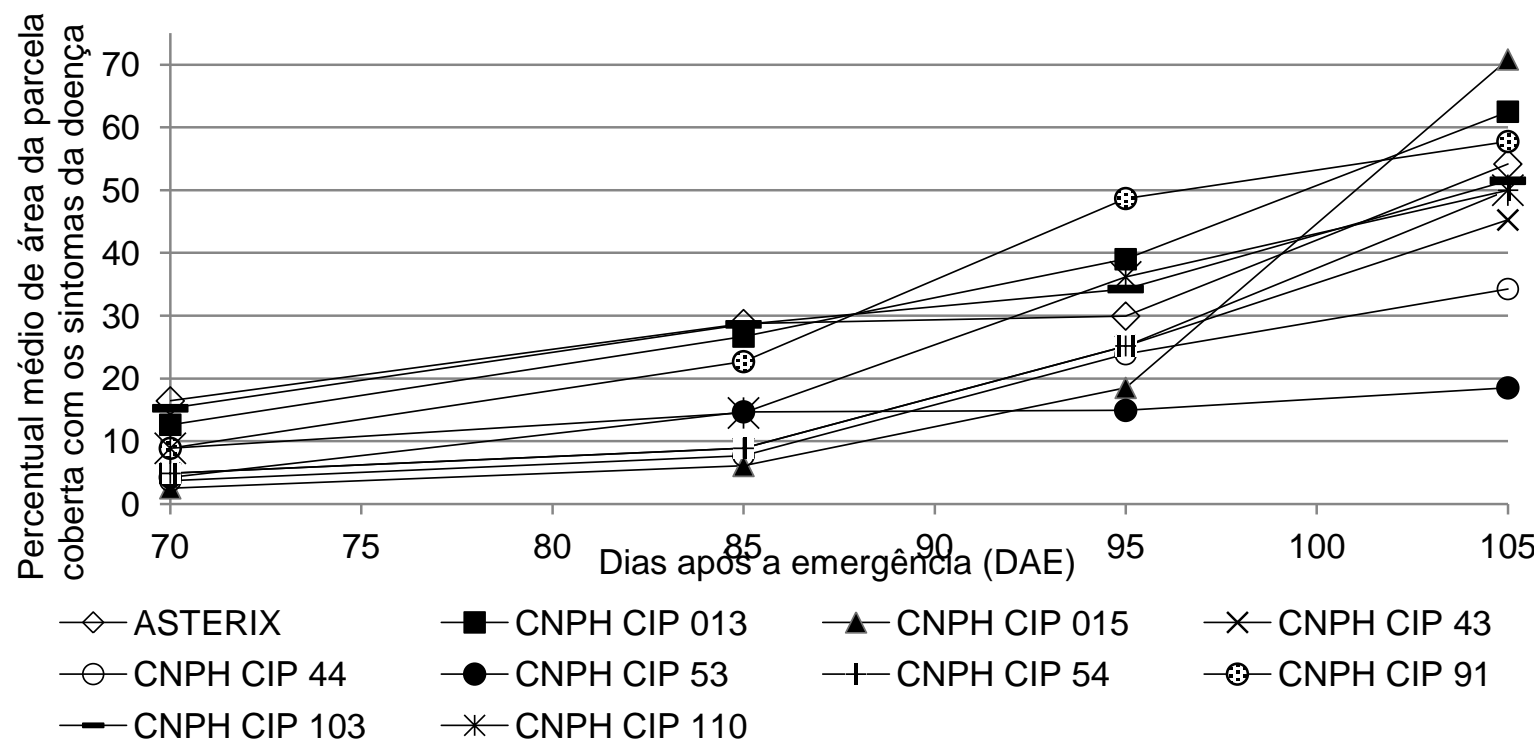

Figura 1 - Percentual médio de área da parcela coberta com os sintomas de pinta-preta a partir dos 70 até os 105 dias após a emergência (DAE). Average percentage of area of plot covered with symptoms of early blight from 70 to 105 days after emergence (DAE).

O maior destaque deve ser dado ao clone CNPH CIP 53, que obteve índices bem inferiores de severidade (Figura 1), sendo estes constantes ao longo de todo o ciclo, sugerindo, assim, bom nível de resistência à pinta-preta, além de estabilidade. Segundo PINTO et al. (2002), o comportamento de uma determinada cultivar deve ser constante durante todo o ciclo, 
isto é, se uma cultivar for identificada como resistente na avaliação, aos 60 dias após plantio, é de extrema importância que ela mantenha seu desempenho nas avaliações subsequentes. Tal evento é notório para o clone CNPH CIP 53 (Figura 1).

Segundo SILLERO \& RUBIALES (2002), a quantificação dos componentes da resistência permite a separação de genótipos em classes dentro da resistência e, ainda, pode sugerir possíveis mecanismos de resistência. Assim, um dos componentes para a análise da resistência é a severidade da doença, a qual pode ser mensurada pelo percentual de área foliar com tecido necrosado e, geralmente, é estimada utilizando uma escala descritiva ou diagramática, conhecida e desenvolvida para a doença em questão (AGRIOS, 2005). Segundo PAULA \& OLIVEIRA (2003), o uso de severidade de doença, na avaliação de intensidade de doenças causadoras de manchas foliares, provavelmente, é mais adequado. Deste modo, foi possível aferir a severidade de pinta-preta a partir de avaliações aos 70; 85; 95 e 105 DAE, utilizando-se de uma escala descritiva de notas adaptada de REIFSCHNEIDER et al. (1984).

No presente estudo, a cultivar Asterix, considerada resistente e de ciclo longo, foi comparada com outros genótipos. Entretanto, esta não foi mais resistente à pinta-preta do que os clones CNPH CIP 53 e CNPH CIP 44. Similarmente, ROSSI et al. (2011) verificaram que a cultivar Asterix foi severamente infectada pela pinta-preta. Uma possível explicação para estes eventos pode ser o fato de que a pinta-preta pode tornar-se muito destrutiva quando em condições de alta temperatura (25 a $30^{\circ} \mathrm{C}$ ), e a umidade relativa do ar próxima a 90\% (MAFFIA et al., 1980). Assim, um grande desafio para o melhoramento de plantas, visando à resistência a doenças, é a manutenção da resistência quando este material é submetido a diferentes condições de temperatura, localidade geográfica e variações na intensidade de doença, havendo assim a necessidade de testes posteriores em diferentes localidades.

\section{Conclusões}

1- Os clones CNPH CIP 53 e CNPH CIP 44 são os melhores clones quanto à resistência à pinta-preta.

2 - Modelos de regressão linear são bem ajustados para estimar a taxa de progresso da pinta-preta da batata a partir dos 70 DAE, pois são significativos e com alto valor de coeficiente de determinação $\left(R^{2}\right)$.

\section{Agradecimentos}

Os autores agradecem à Fundação de Amparo à Pesquisa do Estado de Goiás (FAPEG) por uma bolsa de mestrado e à Universidade Estadual de Goiás (UEG) pelo suporte financeiro.

\section{Referências}

AGRIOS, G. N. Plant Pathology. $5^{\text {th }}$ ed. Oxford: Academic Press Publications, 2005. 922p.

DITA RODRIGUEZ, M. A.; BROMMONSCHENKEL, S. H.; MATSUOKA, K.; MIZUBUTI, E. S. G. Components of resistance to Early Blight in four potato cultivars: Effect of leaf position. Journal of Phytopathology, Oxford, v.154, n.4, p.230-235, 2006.

FAO - Food and Agriculture Organization of the United.Disponível

em:<http://faostat.fao.org/site/562/default.aspx>. Acesso em: 27 Ago. 2013.

FERREIRA, D. F. Sisvar: a computer statistical analysis system. Ciência e Agrotecnologia, Lavras, v.35, n.6, p.1039-1042, 2011.

FILGUEIRA, F. A. R. Novo manual de Olericultura. 3. ed. Viçosa: Editora UFV, 2008. p.161194.

IBGE - Instituto Brasileiro de Geografia e Estatística. Indicadores IBGE: estatísticas da produção agrícola. Mar. de 2013. 79p. Disponivel em: $<$ http://www.ibge.gov.br/home/estatistica/indicad ores/agropecuaria/lspa/estProdAgr 201303.pdf>. Acesso em: 27 ago. 2013.

JANSKY, S. H.; SIMON, R.; SPOONER, D. M. A test of taxonomic predictivity: Resistance to Early Blight in wild relatives of cultivated potato. Phytopathology, Saint Paul, v.98, n.6, p.680687, 2008.

MAFFIA, L. A.; MARTINS, M. C. P.; MATSUOKA, K. Doenças do tomateiro. Informe Agropecuário, Belo Horizonte, v.6, n.66, p.42-60, 1980.

MAGGIO, A.; CARILLO, P.; BULMETTI, G. S.; FUGGI, A.; BARBIERI, G.; PASCALE, S. Potato yield and metabolic profiling under conventional and organic farming. European Journal of Agronomy, Amsterdam, v.28, n.3, p.343-350, 2008.

NEDER, D. G.; PINTO, C. A. B. P.; MELO, D. S.; LEPREIV, A. L.; PEIXOUTO, L. S. Selection of potato clones for multiple resistances to early blight, PVY and PVX. Ciência Rural, Santa Maria, v.40, n.8, p.1702-1708, 2010.

PAULA, R. S.; OLIVEIRA, W. F. Resistance of tomato (Lycopersicon esculentum) to Alternaria 
solani pathogen. Pesquisa Agropecuária Tropical, Goiânia, v.33, n.3, p.89-95, 2003.

PEIXOTO, N.; FILGUEIRA, F. A. R.; MELO, P. E.; BUSO, J. A.; MONTEIRO, J. D.; BRAZ, L. T.; PURQUERIO, L. F. V.; HAMASAKI, R. I. Selection of potato cultivars for high altitude microclimates in Central Brazil. Horticultura Brasileira, Brasília, v.20, n.3, p.438-441, 2002.

PINTO, C. A. B. P; FARIA, C. A.; LAMBERT, E. S. Potato clones resistance to early and late blight. Crop Breeding and Applied Biotechnology, Viçosa, MG, v.2, p.189-196, 2002.

PIMENTEL GOMES, F. Curso de estatística experimental. 14. ed. Piracicaba: Degaspari, 2000. 477p.

REIFSCHNEIDER, F. J. B; FUROMOTO, O.; FILGUEIRA, F. A. R. Illustrated key for the evaluation of early blight of potatoes. Plant Protection Bulletin, Taiwan, v.32, n.3, p.91-94, 1984.

RODRÍGUEZ, N. V.; KOWALSK, B.; RODRÍGUEZ, L. G.; CARABALlOSO, I. B.; SUÁREZ, M. A.; PÉREZ, P. O.; QUINTANA, C. R.; GONZÁLEZ, N.; RAMOS, R. Q. In vitro and ex vitro Selection of Potato Plantlets for Resistance to Early Blight. Journal of Phytopathology, Oxford, v.155, n.10, p.582-586, 2007.
ROSSI, F.; MELO, P. C. T.; AZEVEDO FILHO, J. A.; AMBROSANO, E. J.; GUIRADO, N.; SCHAMMASS, E. A.; CAMARGO, L. F. Potato cultivars for organic production systems. Horticultura Brasileira, Brasília, v.29, n.3, p.372-376, 2011.

SHAHBAZI, H.; AMINIAN, H.; SAHEBANI, N.; HALTERMAN, D. Effect of Alternaria solani exudates on resistant and susceptible potato cultivars from two different pathogen isolates. The Plant Pathology Journal, Suwon, v.27, n.1, p.14-19, 2011.

SILLERO, J. C.; RUBIALES, D. Histological characterization of resistance to Uromyces viciae-fabae in faba bean. Phytopathology, Saint Paul, v.92, n.3, p.294-299, 2002.

SIMON, G. A.; PINTO, C. A. B. P.; LAMBERT, E. S.; ANDREU, M. A. Potato clones selection for early blight resistance and heat tolerance. Revista Ceres, Viçosa, MG, v.56, n.1, p.031037, 2009.

TÖFOLI, J. G.; MELO, P. C. T.; DOMINGUES, R. J.; FERRARI, J. T. Potato late blight and early blight: importancy, characteristics and sustainable management. Biológico, São Paulo, v.75, n.1, p.33-40, 2013. 